\title{
1968 MEMBERSHIP DIRECTORY
}

RUTH L. AASKOV, Dir. Lang Lab., Augsburg College, Minneapolis, Minn. 55404 JOHN A. AHERN, Lang. Lab. Dir., 87 Madison Ave., Valhalla, N. Y. 10595 CHRISTA AHRENS, Lang. Lab. Dir., 33 Gilmer St. SE, Box 92. Atlanta, Georgia 30303 FAROUK B. ALAMEDDINE, Supv. Lang Lab., Wayne State Univ., 308 State Hall, Detroit, VIRGINIA BROWN ALIOTTI, Box X, Madison College, Harrisonburg, Va. 22801 KENTON H. ALLEN, Dist. Lang. Coord., 2100 W. 55th St. So., Wichita, Kansas 67217 ROBERT F. ALLEN, Dir. Lang Lab., Rutgers St. Univ., Lang Bldg., New Brunswick, N. J. BETHEL AMARO, Supv. English Section, Dept. of Education, Hato Rey Puerto Rico LOU AMAYA, Asst. Prof. of For. Lang., Tarkio College, Tarkio, Missouri 64491 C. J. AMBERT, Lang. Lab. Dir., East Carolina Univ., Greenville, N. C. 27834 PROF. ANDREWS, Head Rom. \& Class Lang., Univ. of Connecticut, Storrs, Conn. 06268 P. C. Annable, Chrm., Slippery Rock St. College, Slippery Rock, Penn. 16057

I. C. ARENSMAN, Chrm. For. Lang., Metropolis Comm. H. S., 1004 Catherine St, Metropolis, III 62960

JOANNA ARMSTRONG, Dir. Lang. Lab., Texas Southern Univ., Houston, Texas 77004

KATHRYN ARNOLD, Dir. Lang. Lab., Adelphi Univ., Garden City, N. Y. 11530

ERNEST E. ASHLEY, Lang. Lab. Supv., Bemidji St. College, Bemidji, Minnesota 56601

CECIL ATTIG, Supv. California St. College at Fullerton, 800 N. St. Col. Blvd., Fullerton, Calif. MARGareT M. ATWOOD, Chrm. Mod. Lang., Husson College, Patk St., Bangor, Me. 04401 GILLES E. AWGER, Dir. Lang. Lab., Nasson College, Springvale, Maine 04083

DR. L. E. BAAK, Chrm. Dept. Mod. Lang., Morningside College, Sioux City, Iowa 51106 REID E. BAHER, Lang. Lab. Dir., Allegheny College, Neadville, Pa. 16335

VIVIJA N. BALTINS, Inst., Lang. Lab. Asst., St. Francis College, Fort Wayne, Ind. 46808 PEDRO BARRIO, Prof. of Spanish, Converse College, Spartanburg, S. C. 29301 REV. GILBERT BARTH, Chrm. Dept. of Mod. Lang., Col, of Steubenville, Steubenville, Ohio RALPH W. BASSETT, Supv. For. Lang., Portland Pub. Sch., P. O. Box 3107, Portland, Ore. HUGO J. BAUER, Dir. AV \& Lang. Lab., Lindenwood College, St. Charles, Mo. 63301 KEITH R. BELL, Inst. of Spanish, 23 E. Elm St., Wollaston, Mass. 02170

SISTER M. BERNYNE, Asst. Prof. of Fr.., Viterbo Col. 815 S. 9th St., LaCrosse, Wis. 54601 SAMUEL BERR, Lang. Lab. Dir., St. Univ. of N. Y., Dept. of German, Stony Brook, N. Y. PIERRE BIARNES, Lang. Lab. Dir., Macomb Comm. Col., 1450012 Mile Rd., Warren, Mich. GILleS BIBEAU, Chef des Lab de Langues, Universite de Montreal, C. P. 6128, Montreal 3, Quebec, Canada

PAMELA BLACK, Supv. Lang. Lab., Mt. Allison Univ., Sackville, N. D.

JOSEPH C. BLAIR, Lang. Lab. Dir., Univ. of Missouri, Lang. Lab. A \& S 39, Columbia, Mo. CHARLES H. BLATCHFORD, 509 W. 110 St. Apt. SA, New York, N. Y. 10025

JAMES P. BLOUNT, Dir. Lang. Lab., William \& Mary Col., Williamsburg, Va. 23185 HANS BODLANDER, Inst. Lang. Lab., Univ. of Bridgeport. Dana Hall, Bridgeport, Conn.

BROTHER JAMES BONILLA, Chrm. Mod. Lang. Dept., LaSalle Military Acad.,Oakdale, L. I. EDWARD F. BOOTH, St. Supv. Mod. Lang. St. of Me., Dept. of Ed., Augusta, Me., 04330 ROBERT O. BOOTHE, Lang. Lab. Dir., Calif. St. Polytech. Col., San Luis Obispo, Calif. 93401 WARREN C. BORN, Chrm. For. Lang. Ed., N. Syracuse Central Sch., Andruson Center, 201 S. Main St., North Syracuse, N. Y. 13212

HERBERT BOSWAN, Lang. Lab. Supv., Univ. of N. D., Box 8275 Univ. Station, Grand Forks, N. D. 58201

ROGER GOUGARD, Dir. Lang. Lab., N. C. College, Box 609, Durham, N. C. 27707

FERNAND C. BOURASSA, Lang. Lab. Dir., Calgary Pub. Sch. Brd., 111 Grafton Dr., S. W., Calgary Alberta, Canada

LANSDON H. BOWEN, Dept. For. Lang., Thiel College, Greenville, Penn. 16125

PATRIC C. BOYDEN, Dir. Lang. Lab., Kent St. Univ., Kent, Ohio 44240

LEE E. BRANCH, Dir. Lang. Lab., Mason Hall Rm. 212, Paloma Col. Claremont, Calif. 91711 LEON E. BRAXTON, Lang. Lab. Dir., Stetson Univ., Box 1375, Deland, Fla. 37200

FE C. BRITTAIN, Inst., W. Chicago Comm. H. S., Joliet St., West Chicago, Ill. 60185

ERIKA S. BROSSMAN, Asst. Dir. Mod. Lang. Lab., Albright College, Reading Penn. 19604

DR. LUTHER F. BROSSMAN, Dir. Mod. Lang. Lab., Albright College, Reading, Penn. 19604 S. RAY BROST, Asst. Prof., Ger. Dept., Lycoming College, Williamsport, Penn.

EL.DON I. BROWN, Lang. Lab. Dir., Springfield Jr. College, 1500 N. Fifth St., Springfield, Ill. WILBUR J. BRUNER, Willinm Jewell College, Liberty, Mo. 64068 
LAWRENCE BRUNK, Instr. Sp., Bluffton College, Bluffton, Ohio 45817

SISTER M. ALBAN BSHARAH, Dir. Lang. Lab., Col. of New Rochelle, New Rochelle, N. Y. DR. GEORGE C. BUCK, Dir. Lang. Lab., U. of Washington, 118 Denny Hall, Seatcle, Wash. SR. MARY M. BUELT, Inst. in French, Fontbonne Col., 6800 Wydown Blvd., St. Louis, Mo. Magdelhayne BUTEaU, Chrm. Fr. Dept., St. Joseph Col., 3465 Durocher St., Montreal Quebec, Canada

J. W. BUTLER, Lang. Lab. Tech. Dir.. Univ. of Mass., Bartlett Hall, Amherst, Mass. 01003 A. L. BUZZARD, Asst. Prof., College of Idaho, Caldwell, Idaho 83605

ANTHONY A. CACOSSA, Asst. Prof. Mod. Lang., Towson St. Col., Baltimore, Md. 21204 DR. ROMAN DE LA CAMPA, Chrm., Mod. For. Lang., Briar Cliff Col., Sioux City, Iowa PIERRE J. CAPRETZ, Dir. Lang. Lab., Yale Univ., 111 Grove St., New Haven, Conn. 06510 FLORENCE CARLSON, Lang. Lab. Supv., Wellesley College, Wellesley Mass. 02181 EMORY W. CARR, Inst., Marshall Univ., P. O. Box 114, Huntington, W. Va. 25701

SR. MARY CATHERINE OP, Chrm. For. Lang., Edgewood College of Sacred Heart, 855 Woodrow St., Madison, Wis. 53711

CHRISTINE E. CERVIZZI, Lang. Coord., Northbridge H. S., Linwood Ave., Whitinsville, Mass. JANE SCOTT CHAMBERLAIN, 6416 Winnepeg Road, Bethesda, Maryland 20034

DAVID G. CHANDLER, St. Olaf College, Northfield, Minnesota 55057

SISTER JANE DE CHANTAL, Chrm. For. Lang. Dept., Frontbonne Col., 6800 Wydown, St. Louis Mo. 63105

DR. R. CharbonneaU, Dir. Dept. of Lang., Univ. de Montreal, C. P. 6128, Montreal 3, Quebec, Canada

SISTER MARY CHARLOTTE, Marywood H. S., 2128 Ridge Ave., Evanston, III. 60201

LOUIS J. ChATAGNIER, Lang. Lab. Dir., Emory Univ., Box 152G3, Atlanta, Ga. 30333

HENRY R. CHRISTMAN, Dir. Lang. Lab., Kutztown St. Col., 215 Wellington Ave., Lincoln Park, Reading, Penn. 19609

A. J. CICERAN, Lang. Lab. Supv. Brock Univ., St. Catharines, Ontario

ARTHUR CICERO, Dir. Lang. Lab., St. Vincent College, Latrobe, Penn. 15650

EUGENE CLAIR, Dir. Lang. Lab., Franklin \& Marshall Col., Box 24, Lancaster, Penn. 17604

BEN T. CLARK, Instr., 674 Amesti Rd., Cabrillo College, Watsonville. Calif. 95076

PATRICK R. ClARKE, Lang. Lab. Dir., Bradford Jr. Colege, Bradford, Mass. 01832

PROF. CALVIN ClAUdel, Chrm. Dept. of Rom. Lang., W. Va. Wesleyan Col., Box 98, Buckbannon, W. Va. 26201

RUTH W. COLUMBO, Lang. Dept. Head, Natick H. S., 15 W. St., Natick Mass. 01760

JEAN S. CONKEY, Supv. Lang. Lab., Wheaton College, Norton. Mass. 02766

DR. MARIL YN J. CONWEll, Lang. Coord., Penn St. Univ., 3421 West Penn St., Philadelphia, Penn. 19129

DR. D. CHAVY COOPER, Chrm. Lang. Dept., Keuka College, Keuka Park, N. Y. 14478

HENRY F. COOPER, Dir, Lang. Labs., Univ. of Oregon, Rom. Lang. Dept., Eugene, Oregon PAUL J. COOPER, Dir. Lang. Lab., Univ. of Tenn., Knoxville, Tennessee 37916

DR. JAMES E. COPELAND, Dir. Lang. Dept., Rice Univ., P. O. Box 1892, Houston, Tex. BR. H. MICHAEL CORRY, FSC, Lang. Lab. Dir., Christian Brothers Academy, Lincroft, N. J. RONALD KENT COSTA, Inst. French \& German, 20 Greenwood St. Oakhill, Newton, Me.

NORMAN R. COTE, Head For. Lang. Dept., N. Shore Comm. Col., 3 Essex St., Beverly, Mass. EARL F. COUEY, Lang. Lab. Supv., Sonoma St. Col., 1800 E. Cotati Ave., Rohnert Park, Calif. MADeleine A. COURNOYER, Chrm. For. Lang. Dept., Proviso E H. S., First Ave. \& Madison, Maywood, III. 60153

MARTIN J. COYNE JR., Tech. Asst., Univ. of Mass., Boston, 100 Arlington St., Boston, Mass. MARY NUTE CRAIGHILL, Tape Librarian, Harvard Univ., Mod. Lang. Cntr, Cambridge, Mass. ROSEMARY CRIPPEN, Lang. Lab. Dir., Fenton H. S., 404 W. Ellen St., Fenton, Mich. 48430 CARLOS V. CRUZ, Lang. Lab. Supv., Univ, of Rochester, Fauver Hall Rm. 212, River Campus. Rochester, N. Y. 14627

ROBERT A. CULlinS, Chrm. For. Lang., R. E. Lee H. S., Neely \& Tarleton St., Midland, Tex. LEO H. DANIELS, Marietta College, Box 56, Marietta, Ohio 45750

REV. RAFAel R. DAVILA MM, Dir. Lang. Lab., Maryknoll Seminary, Glen Ellyn, Ill. 60137 ROSEMARY DAVIS, 502 Huron Ave., Cambridge, Mass.

MRS. J. DelanOeYE, Lan. Lab. Dir., Calif. St. Col., at Los Angeles, 5151 St. Col. Fr., Los Angeles, Calif. 90032

Dept. of For. Lang., Rhode Island Col., 600 Mt. Pleasant Ave., Providence, R. I. 02908

Dept. of Linguistics, Univ. of Calif., San Diego, La Jolla, Calif. 92038 
DR. LARRY R. DEROULEN, Lab. Dir., Univ. of S. W. La., Dept. For. Lang. USL, Lafayette MR. EMILIO DE TORRE, Lang. Lab. Dir., Manhattanville Col., of the Sacred Head, Purchase, N. Y. 10577

JUSTIN M. DEVLIN, Head MFL Dept., Lawrence H. S., Lawrence, Mass 01840

DIEGO DE VARGAS, For. Lang. Coor., Carlsbad Municipal Sch., 103 W. Hagerman St., Carlsbad, N. M. 88220

ELMER DIETRICH, Head Lang. Dept., Miller H. S., Miller, S. D. 57362

Dir. of Lang. Lab., Nasson College, Springville, Maine 04083

VITA J. DI VINCEZA, Spring \& Hilldale Rd., Malvern Rd. 2, Penn. 19355

Division of Humanities, Lang. Dept., 2140 Po St., Tuskegee Institute Ala. 36088

JAMES W. DODGE, Tech. Dir. Lang. Lab., Brown Univ., Box E. Marston Hall, Providence

PIERRE R. DUCRETET, Asst. Lang. Lab. Dir., Univ. Col., Univ, of Toronto, Toronto 5,

Ontario, Canada

JOHN D. DURDEN, 4738 Everhart Dr., North Canton, Ohio 44720

W. M. ECHOL.S, Lang. Lab. Supv., Emory Univ., 1500 Pierce Dr. Apt. 105, Atlanta, Ga. 30307 E. J. EILERTSON, 414 W. Park Ave., Waukesha, Wis. 53186

BRO. A. D. EISCH, Lang. Lab. Dir., Catholic Memorial H. S., 235 Baker St., West Roxbury, Mass. 02132

RALPH A. EISENSTADT, Lang. Lab. Dir., For. Lang. Dept. West Chester St. Col., West Chester, Penn. 19380

MRS. JANE R. ELDON, Lang. Lab. Dir., North Central Col., Naperville, IIl. 60540

MRS. LUCY ELIFSON, Lang. Lab. Dir., Waukesha H. S., 400 N. Grand Ave., Waukesha, Wis. WILliaM F. ELMENDORF, Asst. Prof., Western Washington St. Col., Bellingham, Wash.

MAURICE G. ELTON, Dir. Lang. Lab., S. Methodist Univ., Clements Hall, Dallas, Tex. 75222 PAUL, EMOND, Lang. Lab. Dir., Ecole St. Andre, Lasarre, Quebec Canada

CARL T. ERICKSON, Asst. Prof., Univ. of Alberta Calgary, Dept. of Mod. Lang., Calgary, Alberta Canada

LORENZO ESPANOSA, Supv. Lang. Lab., Univ. Calif., Riverside, Calif. 92502

WILliaM V. EWALD, Lang. Lab. Dir., Concordia Tea. Col., Box 9-C. 7400 Augusta River, Forest, Ill. 60305

MARILYN AYN FANALY, Hd. Lang. Dept., Hesperia Sr. H. S., Hesperia, Mich. 49421

JAMES C. FAULKNER, Div. Chrm., St. Univ. Col., New Paltz, N. Y. 12561

MARTIN A. FAVATA, Chrm., Dept. For. Lang., Tenn. Technological Univ., Cookerville, Tenn. PERCY FEARING, MFL Consultant, St. Dept. of Ed., Centennial Office Bldg.. St. Paul, Minn. LEO J. FECTEAU, Head F/L Dept., Barrington Pub. Sch., Barrington H. S., Barrington, R. I. ALVIN FeldmaN, For. Lang. Coord., Sch. Dist. No. 3, P.O. Box 1500, Huntington, N. Y. JOHN W. FERGUSON, Lang. Lab. Dir., Murray St. Univ., Box 56, Murray, Kentucky 42071 DR. AUGUSTINE FERNANDEZ, Asst. Prof. Rom. Lang., Bishop Col., 3837 Simpson Stuart, Dallas, Tex. 75241

DR. JOSE C. FERNANDEZ, Assoc. Prof. Sp. St. Univ. Col., 6 Lakeside Ct., Plattsburgh, N. Y. MRS. NINA FERSON, Dir. Lang. Lab., Williams College, Williamstown, Mass. 01267 BRUCE L. FINK, 296 Ohio St., Pasadena, Calif. 91106

C. BRUCE FITCH, Lang. Lab. Dir., Transylvania Col, 300 N. Broadway, Lexington, Ky. 40508 JULIA FlEMMING, Dir. Lang. Lab., 709 N. Washington St., Royal Oak, Mich. 48067

N. H. FORBES, Dir. Lang. Lab., Waynesboro H. S., 1200 W. Main St., Waynesboro, Va. JOHN EL.DON FORD, 213 2nd St. East, Ashland, Wis. 54806

FOREIGN LANG. DEPT., Fresno St. College, Fresno, Calif. 93726

JAMES F. FOURNET, Dir. Lang. Lab., S. E. Louisiana Col., 1004 So. Oak St., Hammond, La. ROBERT FOURNIER, Consit. For. Lang., St. Dept. of Ed., 410 St. House Annex, Concord, N. H. 03301

BETTY FOWLER, For. Lang. Consult., Stanislaus Co. Schls., P. O. Box 1697, Modesto, Calif. ERNEST T. FOX, Dir. Lang. Lab., St. Univ. Col., Geneseo St. Univ. Col., Geneseo, N. Y. RAYMOND A. FRAHM, Dir. Lang. Lab., St. Univ. College, Fredonia, N. Y. 14063 DR. MARY L. FRANCIS, Chrm. Rom. Lang. Dept., Salisbury St. College, Salisbury, Md. 21801 EDWIN J. FRANK, Dir. Lang. Labs., Miami-Dade Jr. College, Miami, Fla. 33167

ERNEST A. FRECHETTE, Sr. Supv, Lang., Mass. Dept. of Ed., P. O. Box 98, East Freetown, Mass. 02717

MARGARET FREEMAN, Mass. Inst. of Tech., Room 14N-222, Cambridge, Mass. 02139 HECTOR FRENETTE, Audio Visual Dir., Regional Sch. Bd., P. O. Box 39, Rimouski, Quebec Canada 
DR. REINHOLD FREUDENSTEIN, 3579 Steinatal, Germany

ARTHUR F. FROST, Dir. Lang. Lab., Princeton Univ., 304 E. Pyne Bldg., Princeton, N. J. JOE K. FUGATE, Chrm., Dept. German, Kalamazoo College, Kalamazoo, Mich. 49003

ClarenCe R. FUlleR, Chrm., Dept. Mod. Lang., Bloomficld Col., Bloomfield, N. J. 07003 JOSEPH F. FUSCO, Lang. Lab. Dir., Dept. of Eng., St. Michael's Col., Winooski, Vt. 05404 REV. ULYSSES A. GALVAO, 175 II Rd., Lake Forest, Ill. 60045

ERMEL E. GARINGER, Dir. Lang. Labs., Univ, of Kansas, Lawrence Kansas 66045

PROF. LOIS S. GAUDIN, Brooklyn Col. City Univ. N. Y., Dept. of Mod. Lang., Bedford \&

H. Ave., Brooklyn, N. Y. 11210

ALAN GARFINKEL, Rom. Lang. Dept.. 154 Oval Dr., Columbus, Ohio 43210

THOMAS E. GARST, Dir. Lang. Lab., Civdad Universitaria, Mexico D. F.

WARREN E. GATES, Dept. of For. Lang., Ohio Northern Univ., Ada, Ohio 45810

PAUl GeBaUER, Linfield Col., Box 464, Linfield Col., McMinnville, Oregon 97129

CARL W. GEFFERT, Ger. Dept., St. Andrews College, Laurinburg, N. C. 28352

ANNE H. GeliNeAU, Dir. Lang. Lab., Harvard Univ., G-4 Boylston Hall, Cambridge, Mass.

GeORGe GIANNETTI, Chrm. For Lang., Oak Park H. S., 13701 Oak Park Blvd., Oak Park, Mich. 48237

SUSAN GILBERT, Dir. For. Lang. Lab., Wofford Col., Church St., Spartanburg, S. C. 29301

ZENOBIA GILBERT, Lang. Coord., Sewanhaka H. S., 500 Tulip Ave., Floral Park, N. Y. 11001 ARTHUR J. GIONET, North Texas St. Univ., Denton, Texas

DR. HENRY GLADE, Manchester Col., Box 36, North Manchester, Ind. 46962

E. M. GOLDSTEIN, Dir. Lang. Lab., Memorial Univ, of Newfoundland, St. John's, Newfoundland, Canada

RONALD GOLDSTEIN, Technician, Univ. of Cal. Santa Barbara, Lang Lab., Goleta Calif.

T. R. GOLDSWORTHY, Dir. Lang. Labs, Univ. of Wisconsin, 373A Bascom Hall, Madison JAIME J. GONZALEZ, Dir, For. Lang. Lab., Tusculum Col., P. O, Box 77, Tusculum, Tenn. ALAN M. GORDON, Dept. of Ital. \& Hispanic, Univ. of Toronto, Toronto 5, Ont., Canada IAN B. GORDON, Lang. Lab. Dir., Baptist Col. at Charleston, P. O. Box 10087, Charleston, S. C. 29411

MARTIN E. GOTTSCHALK, Lab. Supv., Howard Payne Col., Brownwood, Tex. 76801

WENDY GRAEF, Dir. Lang. Lab., William Woods Col., Fulton, Mo. 65251

MARY C. GRAY, Lang. Lab. Supv., Lenoiur Rhyne Col., 616 8th St. N.E. Hickory, N. C. 2860 ROBERT B. GREEN JR.. 48 Smithfield St., Buckhannon, W. Va. 26201

ESTELlE S. GREENE, Dir. Lang. Lab., Fairleigh Dickinson Univ., 207 Montross Ave., Rutherford, N. J. 07070

Cl.ARA GREGORY, Const., Texas Education Agency, $3010 \mathrm{~K}$. Windsor Rd., Austin, Tex. 78703 CHARLES GRIBBLE, Instr. Russian Lang. and Com. Lit., Bradeis Univ., Waltham, Mass. 02154 FRANK GRITTNER, For. Lang. Supv., Wis. Dept. Public Instr., B-11 So. Capitol, Madison STEFANIA E. GROSS, Dir. Lang. Lab., Bowling Gm. St. Univ., Univ. Hall, Bowling Grn., O. SISTER ELIZABETH GROSSE, Dir. Lang. Lab., Aluerno Col., 3401 So. 39th St., Milwaukee SISTER M. ANSELM GROVER, Lang. Lab. Dir., College Holy Names, 3500 Mountain Blvd., Oakland, Calif. 94619

NATHANIEL GRUNST, Chrm. For. Lang. Dept., Luther H. S. North 5700 W. Berteau Ave., Chicago, III. 60634

PAUL G. GUENETTE, Dir. For. Lang., Town of Brookline, 333 Washington St., Brookline, Mass. 02146

EDW ARD M. GUGGER, For. Lang. Dept. Head, Medford H. S., Medford, Mass. 02155

ROSALYN GURA, Lang. Lab. Supv., Youngstown St. Univ., 410 Wick Ave., Youngstown, $O$. LYNN O. HAGAN, Div, Head For. Lang., Lee College, Box 30, Baytown, Tex. 77520 HILMAN VON HALEM, Head Lang. Div., 170 Beacon St., Boston, Mass. 02116

ERWIN M. HAMSON, Dir. \& Lect., Univ. of Michigan, 1401 Mason Hall, Ann Arbor, Mich. JACOBINA HARDING, Lang. Lab. Supv., Alamo Hgts., H. S., 6900 Broadway, San Antonio BRO. EDWARD HARRISON, Inst. Walsh Col., 2020 Easton Rd., Canton, Ohio 44720 IRENE HASENCLEVER, Lang. \& Lit. Div., Bennington College, Bennington, Vermont 05201 MISS YUKIKO HAYASE, Dir. Lang. Lab., Winthrop College, Rock Hill, S. C. 29730 EDITH HELMAN, Chrm., Dept. For. Lang., Simmons Col., 300 The Fenway, Boston, Mass. JOAN L. HERNANDEZ, Dir. Lang. Lab., L. S. U. at Eunice, Eunice, Louisiana 70535 MARY N. HERNANDEZ, Technician, Tennessee St. Univ., Box 550, Nashville, Tenn. 37207 DR. RAFAEL A. HERNANDEZ, Virginia Union Univ., 17 Meadow Lark Lane, Richmond, Va. 
LAZARO M. HERRERA, Lang. Lab. Dir., Westminster Sch., 1424 W. Paces Ferry Rd. N. W., Atlanta Georgia 30327

H. READE HESKAMP, Dir. Lang. Lab., MacMurray Col., East State \& Clay, Jacksonville, III. SHARON HEUERMAN, Fr. \& Lab. Supv., Woodstock H. S., Woodstock, III. 60098

DR. J. HEWSON, Memorial Univ, of NFLD, St. Johns Newfoundland, Canada

Charles H. HICKMAN, Lang. Lab. Dir., West Georgia College, Carrolton, Georgia 30117 THOMAS O. HILlER, Fresno State Col., Dept. For. Lang., Fresno, Calif. 93726

MARGARETE HILTS, Chrm. Mod. Lang., La Sierra College, Riverside, Calif. 92505

ALBERT A. HOCH, Lang. Lab. Supv., Lyons Twsp. H. S., 100 So. Brainard, LaGrange, Ill. MARION F. HODAPP, Lang. Lab. Coord., For. Lang. Dept., Col. St. Univ., Ft. Collins, Colo. ROBERT T. HOWLING, Chrm. Eng. \& Humanities, New Haven Col., New Haven, Conn.

DONALD T. HUFFMAN, 781 Meadowbrook Rd., W. Va. Univ., Morgantown, W. Va. 26505 ALEX P. HULL JR., Lang. Lab. Dir., Texas Technological Col., P. O. Box 4285, Lubbock, Tex. HARVEY J. HUMPHREY, Asst. Lang. Lab. Dir., Fordham Univ., Bronx New York, N. Y. HERMAN HURTADO, Comm. Lab. Dir., Florida Atlantic Univ., Boca Raton, Fla. 33432 JOSEPH C. HUTSHINSON, Chief Research \& Studys Div., 502 G St. SW, Washington, D. C. JOHN INGRESOLL, Sr. H. S., Grand Rapids, Minn. 55744

EVELYN UHRHAN IRVING, Lang Lab., Univ, of Guelph, Guelph Ontario, Canada

GORDON JACKSON, Whitman College, Walla Walla, Washington 99362

MARY E. JACKSON, Dir. For. Lang. Lab., Morgan St. Col., Baltimore, Md. 21212

RICHARD L. JACKSON, Asst. Prof., Carleton Univ., Dept. of Sp., Ottawa 1, Ontario Can.

DR. W. RICHARD JACKSON, For. Lang. Coord., Paim Beach County, Bldg. S-502-6th St. N., West Palm Beach, Fla. 33401

J. V. JACOBSON, Lang. Lab. Dir., Centennial Tower Lang. Sch., 400 Laurier W., Ottawa 7, Ontario Canada

FRANCIS G. JARLeTt, Dir. Lang. Lab., Central Conn. St. Col., 265 Wolcott Hill Rd., Wethersfield Conn. 06109

DR. WILLIAM JASSEY, For. Lang. Dir., 105 Main St., Norwalk, Conn. 06851

G. H. F. JOHNSON, Adm. Lang. Lab., Univ. of Brit. Columbia, Vancouver 8, B. C. Canada YUSUKE KAWARABAYASHI, Dir. Lang. Lab., Univ. of Pacific, Stockton, Calif. 95204

ELIZABETH C. KELHOFER, For. Lang. Coord., Chillicothe Sr. H., Yoctangee Parkway, Chillicothe, Ohio 45601

JOSEPH KEMPRECOS, Chrm. For. Lang. Dept., Rumson-Fair Haven Regional H. S., Rumson, N. J. 07760

ELIZABETH C. KENDALL, Ger. Inst., Langston Univ.. Langston Okla. 73050

WILLIAM P. KENEALY, Lang. Lab. Dir., Lexington H. S., 7 Honcock Ave., Lexington, Mass.

CHARLOTTE W. KERR, Supv. Lang. Lab., Occidental Col., Dept. of Lang. \& Ling., 1600 Campus Rd., Los Angeles, Calif. 90041

R. D. KIEKEL, Asst. Prof., Oregon State Unir., Dept. Mod. Lang., Corvallis, Oregon 97331

ARTHUR S. KIMMEL, Audio Labs Coord., Calif. St. Col., at Hayward, 25800 Hillary St., Hayward, Calif. 94542

GEORGE E. KOEHLER, Lang. Lab. Dir., St. Procopius Col., Lisle, Ill. 60532

EGYETEMI KONYVTAR, Sci. Lab., U of Budapest, POB 483, Budapest 5, Hungary

DR. W. LAMARR KOPP, Dir. Lang. Lab., Penn St. Univ., Univ. Park, Penn. 16802

JOHN KOPPENHAVER, Hd. Sp. Dept., Heston College, Heston, Kansas 67062

MR. KOVARY, Lang. Lab. Dir., St. Univ. of N. Y., College at Cortland, Cortiand, N. Y. RICHARD N. KROGH, Arabian American Oil Co., Box 1754, Dhahran, Saudi Arabia

ROY L. KRYNITZ, Lang. Lab. Dir., N. M. St. Univ., For. Lang. Dept., Univ. Park, N M

DR. L. E. KURTH, Chrm. Lang. Lab. Adv. Comm. John Hopkins Univ., 245 Gilman Hall, Baltimore, Maryland 21218

ROBERT LAMBERT, Glenbrook S. H. S., 1700 W. Lake, Glenview, III. 60090

NORMAND J. LAMOUREUX, Lang. Lab. Dir., Holy Cross College, Worcester, Mass. 01610

DAlE L. LANGE, Mod. Lang. Area Head, Univ. H. S., 224 Peik Hall Univ, of Minn., Minneapolis, Minn 55455

BERNARD J. LANGR, Mankato St. College, Mankato, Minn. 56001

LANGUAGE LABORATORIES, Univ. of Texas, Batts Hall 227, Austin Texas 78712

DR. DONALD LAPP, Asst. Prof. of Sp., Aurora College, Aurorn, Ill. 60507

JAMES B. LARKIN, Prof. Sp. Dept., Coe College, Cedar Parso, Iowa 52402 
ELINOR C. LARSON, Dir. Lang. Lab., Scotch Plains-Fanwood H. S., Westfield Rd., Scotch Plains, N. J. 07076

JOSEPH P. LAWLOR, Dir. Lang. Lab., Lake Forest H. S., 1285 N. McKinley Rd., Lake Forest, III. 60045

BRO. FRANCIS LEARY, CSC, Mod. Lang. Dept. Head, Cardinal Mooney H. S., 800 Maiden Lane, Rochester, N. Y. 14615

DAVID LEE, Dept. For. Lang., Fisk Univ., Nashville, Tennessec 37203

E. LEFRANCOIS, Lang. Lab. Dir., Shaw Univ., P. O. Box 79, Raleigh, N. C. 27602

ARTHUR A. LEONE, Lang. Lab. Dir., Indiana Univ. of Penn., Indiana Penn. 15701

MRS. B. LEVINSON, Asst. Lang. Lab. Dir., Brooklyn Col., City Univ., Dept. Mod. Langs., Brooklyn, New York 11210

MARCEL LIDJI, 420 Riverside Dr., New York, N. Y. 10025

GLEN LIEBIG, Lang. Lab. Dir., Bryan College, Dayton, Tenn. 37321

TIMOTHY LIGHT, Asst. Lect., Chinese Univ. of Hong Kong, 6 Farm Rd., Kowtoon Hong Kong

ABRAHAM C. LIN, Asst. Lang Lab. Dir., National Taiwan Univ, Roosevelt Rd., Taipei Taiwan, Republic of China

LUIS LINDNER, Dir. Lang. Lab., Stephens College, Columbia, Mo. 65203

EDGAR A. LIST, Chrm. Dept. For. Lang., Fresno St. College, Fresno, Calif. 93726

MRS. RUTH LOOMIS, For. Lang. Curr. Assoc., Madison Memorial H. S., 201 S. Grammon Rd., Madison, Wis. $\mathbf{5 3 7 0 5}$

DR. A. G. LORE, Dir. Lang. Lab., Univ, N. C., Chapel Hill, N. C. 27514

REBA M. LOWRY, Hd. For. Lang. Dept., Pembroke St. College, Pembroke, N. C. 28372

PAUL F. LUCKAU, Lang. Lab. Dir., Brigham Young Univ., 215 MCK, Provo, Utah 84601

ANNA M. LYNCH, 1325 Dale Dr., Silver Spring, Md. 20910

CLARK LYNN JR., Foreign Lang. Dept., Cameron College, Lawton, Oklahoma 73501

MARY B. MAC DONALD, Lang. Dept. Chrm., Madrid Dependents H. S., Box 908, APO

New York, N. Y. 09283

JOHN MACINKO, Dir. Lang. Labs., Univ. Colo., Old Main Bldg., Boulder Colo 80300

SISTER MARY MADELEINE, College of Notre Dame, 4701 N. Charles St., Baltimote, Md.

SR. MARIE MAGDALEN. Rosary College, River Forest, III. 60305

E. JULES MANDEL, 20918 Calmiali Rd., Woodland Hills, Calif. 91364

G. THOMAS MANN, Inst. Germ., Graceland College, Lamoni, Iowa 50140

SISTER REGINA MARIE OP. Dir. Lang. Lab., Siena Heights College, Adrian, Mich. 49221

CARLOS CORTES MARTINES, Lang. Lab. Dir., Deusto Univ., Apartado de Correos No. 1147, Bilbao-Vizcaya, Spain

DANIEL F. MARTINEZ, Spec. For. Lang., Pueblo Public Sch., 805 Berkley, Pueblo, Colo.

FERNAND MARTY, Hollins College HC. Hollins College, Va. 24020

EDWARD MARXHEIMER, Lang. Lab. Dir., Univ. of Alabama, Rom. Lang., Edmonton, Atla, Canada

ARNOLD H. MARZOLF, Asst. Prof. Germ., N. D. St. Univ., Fargo, North Dakota 58102 MRS. RUTH ANN MAYS, Prof. of French, Pikeville, Ky. 41501

EVELYN MCCLUNEY, Arlington Heights H. S., 4501 W. Rosedale, Ft. Worth, Tex. 76107

DR. J. T. MCCULloUGH, Prof. Sp., Sacramento St. Col., Dept. For. Lang., 6000 J. St., Sacramento, Calif 95819

JERRY MCCUNRU, Lang. Lab., Indiana Univ., P. O. Box 1125, Bloomington, Ind. 47401

JOHN McMULLAN, Dir. Lang. Lab., St. George's Sch., Newport, R. I. 20840

LARRY McWILLIAMS, Coord. For. Lang., Jefferson County Sch. Dist., 1580 Yarrow St., Lakewood, Colo. 80215

CHARLES LEON MEADOWS, Lang. Lab. Dir., Morchouse College, Atlanta, Georgia 30314

FRANK W. MEDLEY JR., Dir. Lang. Dept., Indiana St. Univ., Terre Haute, Ind. 47809

ROY MERCHANT, Ohio Univ., P. O. Box 372, Athens, Ohio 45701

MARY JANE MESS, Chrm. For. Lang. Dept., Ottawa Twnp. H. S., 211 E. Main St., Ottawa, III. 61350

DONALD G. METTLER, Lang. Lab. Dit., Edison Twsp. H. S., S. Colton Rd., Edison, N. J. FLOYD A. MILLER, Commissioner of Ed., 411 S. 13th St., Room 202, Lincoln, Neb. 68509

PROF. ROYCE W. MILLER, Chrm. Lang. Dept., Gordon Col., 255 Grapevine Rd., Wenham, Mass. 01984

E. E. MIRELES, Coord. ABE, CC Public Sch., P. O. Box 1735, Corpus Christi, Tex. 78403

MARLIN M. MITTAG, Chrm. Lang. Dept., East H. S., 910 W. Downer P1., Aurora, Ill. 60506 
MIRCO M. MITROVICH, Chrm. Mod. Lang. Dept., Muskingum Col., 101 Lakeside Dr., New Concord, Ohio 43762

MARY E. MOEN, 11200 Miss. Blyd., Coon Rapids, Minn. 55433

OREN E. MOFFETT, Weber St. College, 3750 Harrison Blvd., Ogden, Utah 84403

TAMARA S. MOISSIY, Dir. Lang. Lab., 10 Mt. Pleasant St., Hyde Park, Mass. 02136

EDITH L. MONSON, Chrm., For. Lang. Dept., Georgetown College, Georgetown, Ky. 40324

DR. J. MICHAEL MOORE, For. Lang. Ed. Spec., San Diego, Bd. Ed., 4100 Normal Ave., San Diego, Calif. 92103

ROBRET L. MORGENROTH, Dir. Lang. Labs., Northern Ill. Univ., 101 Watson Hall, DeKalb, III. 60115

HANES MORRIS, Supy. For. Lang. New Orlcans Pub. Sch., 703 Chrondelet St., New Orleans, La. 70130

ROBERT R. MORRISON, Chrm. Mod. Lang., So. Missionary Col., P. O. Box 475, Collegedale, Tenn. 37315

OLIVIA MUNOZ, Supv. For. Lang., 1300 Capitol Avc., Houston, Tex. 77002

HAROLD T. MURPHY, Asst. Prof. Sp., Marshall Univ., Box 238, 16th St. \& 3rd Ave., Huntington, $\mathrm{W}, \mathrm{Va} .25701$

W. R. MURRAY, Ed. Wiklund Inc., 1216 Springs St. NW, Atlanta, Georgia 30309

KEITH M. MYERS, Dir. Lang. Labs., Univ. III., 214 Lincoln Hall, Urbana, III. 61822

JAMES E. NABERS, Lang. Lab. Dir., South. III. Univ., Dept. For. Langs., Carbondale, III.

MRS. V NAJERA, Faculty LL Supv., NE III. St. Col., 401 Bessely, Elmhurst, IIl. 60126

Nebraska Dept. of Ed., St. Capitol, Lincoln, Nebraska 68509

BERNARD NEWMAN, Lang. Lab. Dir., Bayside H. S., Corn Kennedy St. \& 32nd Ave., Bayside, N. Y. 11361

DR. RICHARD W. NEWMaN, St. Col. at Boston, Place Rd., Boston, Mass. 02115

CHARLES E. NICHOLSON, Consult. MFL, Kansas St. Dept. Pub. Inst., 120 E. 10th, Topeka, Kansas 66612

RUTH A. NIXON, Chrm. For. Lang. Dept., Wisconsin St. Univ., LaCrosse, Wis. $\mathbf{5 4 6 0 1}$

THEODORE NORWICH, Lang. Lab. Dir., W. College for Women, Oxford, Ohio 45056

RICHARD NOVAK, Prof. Chrm. Dept. Concordia Col., 171 White P1. Rd., Bronxville, N. Y. STANLEY J. NUFFer, Lang. Lab. Dir., Box 751, Portland St. Col., Portland, Ore. 97207

MTS. AMPARO B OJEDA, Dir. Lang. Lab., Damen Hall, Rm. 337, N. Sheridan Rd., Loyola Univ., Chicago, Ill. 60626

DR. SVEIN OKSENHOLT, Dept. For. Lang. Chrm. E. Montana Col., Billings, Montana 59101 MORDECAI L. OPHER, Hebrew Teachers Col., 43 Dwight St., Brookline, Mass. 02146.

DR. VIRGINIA OTTO, Chrm. For. Lang. Dept., Nazareth Col. of Rochester, 4245 East Avc., Rochester, N. Y. 14610

DR. S. O. PALLeSKe, Univ. of Denver, Mod. Lang. Dept., Denver, Colo. 80210

CLAUDE A. PAQUETTE, 376 W. Main St., New Britain, Conn. 06052

F. A. PAQUETTE, Executive Sec., ACTFL, 62 5th Ave., New York, N. Y. 10011

MRS. ELAINE PARSONS, Lang. Lab., Green Mountain College. Poultney, Vermont 05764

MR. ARMANDO PARTING, Defense Lang. Inst., US Naval Sta., Navy Annex, Anacostia, Washington, D. C. 20390

DR. RAY PAST, Dept. Eng., Univ. of Texas at El Paso, El Paso, Texas 79999

SISTER MARY PATRIC, OSB, St. Scbolastica H. S., 7416 N. Ridge, Chicago, Ill. 60645

GLORIA A. PATRON, Dir. Lang. Lab. Louisiana St. Univ, at New Orleans, Lakefront, New Orleans, La. 70122

PAUl D. PELOQUIN, Sp. Inst., Chicopee Com. H. S., Rolf Ave., Chicopee Falls, Mass. 01020 REV. R. C. DE PEAUX, Chrm. Dept. Mod. Lang., St. Norbert Col., West De Pere Wis. 54178 RICHARD M. PENTA, For. Lang. Coord., Belmont Sch. System K-12, Belmont, Mass. 02178

PAUl W. PETERSON, Div. Dept. Mod. Lang., Gannon College, Erie, Penn. 16501

D. L. PETHERBRIDGE, Mod. Lang. Supv., Edmonton Pub. Sch. Bd., 11240 79th St., Edmonton Alberta, Canada

ROBERT PHILLIPS, Dir. Lang. Lab., Miami Univ., Oxford, Ohio 45056

PROF. PIERRE L. PINET, Lang. Lab. Dir., Fitchburgh St. Col., Pearl St., Fitchburg, Mass.

PROF. PAUL PIMSLEUR, Dir. Listening Center, Ohio St. Univ., 164 West 17th Ave., Colum. bus, Ohio 43210

GUY PLASTRE, Lang. Lab. Dir., Faculte des Lettes, Universite Laval, Quebec, Canada

VIRGIL L. POULTER, Lang. Lab. Dir., Arlington St. College, Arlington, Tex. 76010

S. JACQUELINE POWERS, Lang. Lab. Dir., Mundelein Col., 6363 Sheridan Rd., Chicago, Ill. 
DR. F. L. PRESTON, Assoc. Prof., Denison Univ., Box 267, Grnaville, Ohio 43023

SISTER M. PROTASE, Dir. Lang. Lab., Col., St. Francis, 500 Wilcox St., Joliet, III. 60435 DANIEL L. RACINE, Lang. Instr., Howard Univ., Fourth St. NW, W/ashington, D. C. 20009 WINSTON J. RESSE, Lang. Lab. Dir., Univ. Iowa, 125 Schaeffer Hall, Iowa City, Iowa 52240 HARRY REGENSTREIF, Lang. Coord., 1220 Wells St., Ann Arbor, Michigan 48104

L. T. C. HOWARD REINDER, Assoc. Prof., US Milita y Academy, Dept. of Lang. USMA, West Point, N. Y. 10996

DON REINKE, Dir. Lang. Lab. Beloit College, Beloit, Wis. 53511

SISTER ROSE RENDAN, 11300 N. E. Second Ave., Miami, Fla. 33161

LILliaN REZBERG, Assoc. Prof. Sp., Bethel College, 1480 No. Snelling, St. Paul, Minn. 55101 DONALD G. RICHARD, St. George H. S., 250 Sherman Ave., Evanston, Ill. 60202

C. P. RICHARDSON, Dir. Lang. Lab., Ohio University, Athens, Ohio 45701

MANFRED RICHTER, Lang. Lab. Dir., Univ. Waterloo, Dept. Germ. \& Russ., Waterloo, Ontario, Canada

DR. A. R. RIEP, Wartburg College, Waverly, Iowa 50677

PATRICIA RIEPER, Prof. Germ., Nebraska Wesleyan Univ., Univ. Park, Lincoln Neb. 68504 ROBERT RIGGS, Shoreline Sch. Dist. No. 412, N. E. 158th \& 20th N. E., Seattle, Wash. 89155 ALBerT RIUSECH, Asst. Prof. Lang., Henderson St. Col., Box 391, Arkadelphis, Ark. 71923 MRS. SUSANNA M. RODRIGUEZ, Long Island Univ., Zeckendorf Campus, Brooklyn, N. Y. R. MAX ROGERS, Brigham Young Univ., 215 McKay Bldg., Provo, Utah 84601

WIBERT J. ROGET, Box 143, Alcorn College, Lorman. Miss. 39096

HELEN RONAN, Dir. Lang. Lab., Washington H. S., Sioux Falls, S. D. 57101

DR. JUSTUS ROSENBERG, Dir. Lang. Ct., Nanyang University, Singapore

S. M. ROSENDA, DML, Coord. Lang. Lab., Regis College, Weston, Mass. 02193

J. ROSSETTI, Wilbur Wright Campus, Chicago City College, 3400 N. Austin Ave., Chicago, Ill. PHILIP ROVNER, Dir. Lang. Lab., Univ. Maryland, College Park, Md. 20742

KATHLEEN KAY RUBIN, Lang. Lab. Dir., Regis Col., W. 50th Ave., \& Lowell Blvd., Denver, Colo. 80221

DR. BARBARA RUCH, Dir. Lang. Lab., 320 Univ. Punn., Philadelphia, Penn. 19104

NORMA RUSCH, Lang. Dept. Head, Lakeland H. S., Minocqua, Wis. $\mathbf{5 4 5 4 8}$

A. J. RYAN, Lang. Lab. Dir., Univ. Mass., Boston, 100 Arlington St., Boston, Mass. 02116 CECIL SACHER, Chrm. For. Lang. Dept., Niles Twp. H. S. North, 9800 Lawler Ave., Skokie, III. 60076

LONNIE SADBURY,, Prof. P. O. Box 788, Grambling. La., 71245

VICTOR SAMPON, Dir. Lang. Lab., Wayland Academy, Beaver Dam, Wis. 53916 BEN D. SANDIDGE, Asst. Prof., Longwood College, Farmville, Va. 23901

PETER D. SANDIER, Consit., Mod. Lang., St. Dept. Education, Montpelier, Vermont 05602 R. EDWARD F. SARNO, Ohio University, Athens, Ohio 45701

JESSE O. SAWYER, Dir. Lang. Lab., Univ. Calif. at Berkeley, 2555 Leimert Blvd., Oakland, Calif. 94602

MRS. WILLIAM SAWYER, 19219 Euclid Avc., Euclid, Ohio 44117

DR. GUENTER G. SCHMALZ, Head Dept. Mod. Lang., Montata St. Univ., Bozeman, Mont. VICTORIA SCHMITZ, Dir. Lang. Lab., Ohio Northern Univ., Ada, Ohio 45810

EBERHARD SCHOLTIS, Asst. Prof. Lang. Instr., Plattsburgh St. Univ. Col., Plattsburgh, N. Y. PROF. MARGARET SCHUDDAKOPF, Dept. of French, Texas College, Tyler,Texas 75701

MILDRED SCHUH, Chrm. Lang. \& Lit. Div., 2405 College Way, Mt. Vernon, Wash. 98273

DAVID SCHULTZ, Supv. Lang. Lab., St. Univ. N. Y. Maritime Col., Ft. Schuyler, Bronx,

N. Y. 10465

DENNIS M. SCHWANK, Inst. Fr. \& Germ., Univ. Minn. at Morris, Morris, Minn. 56267

MAX J. SCHROECK, Blackburn College, Carlinville, Ill. 62626

DOUGlaS SCHWENKER, Lang. Lab. Mgr., Univ. Guelph, Guelph, Ont., Canada

RUSSEL H. SEARCH, Dir. AV Materials, Bucknell Univ., Lewisburg, Pa. 17837

JOHANNES SEIDEL, Lang. Lab. Supv., Rm. 218 Ed. Arts Bldg., Univ. Victoria, Victoria British Columbia, Canada

BETTIE ShelOR, For. Lang. Supv., Pineless Co. Bd. Pub. Inst., 1960 E. Druid Rd., Clearwater, Fla. 33517

EDWIN D. SHAKE, Dir. Lang. Lab., Box 3042 SFA Station, Austin St. Col., Nacogdoches, Tex. 75961

MARY C. SHAPIRO, Asst. Prof., Bridgewater St. College, Bridgewater, Mass, 02320

A. G. SHAW, Head Rom. Lang., Columbia Col., Columbia, S. C. 
MARY M. SHIREY, Supv. Mod. For. Langs., Kanawha County Sch., 200 Elizabeth St., Charleston, W. Va. 25311

LOREN W. SHORES, Chrm. F. L. Dept., Scarsdale H. S., Post Rd., Scarsdale, N. Y. 10583

E. H. SHREFFLeR, Asst. Prof. For. Lang., Lab Dir., Austin Col., Sherman, Texas 75091

DOUGlaS J. SIMMONS, For. Lang. Dept., Arizona St. Univ., Tempe, Arizona 85281

HOWARD L. SIMMONS, 30 Plaza Sq. Apt. 705, St. Louis, Mo. 63103

MERLIN R. SIZELOVE, Chrm. Sp. Dept., Friends Univ., Wichita, Kansas 67213

PETER SKANSE, Dir. Lang. Lab., Wheaton College, Wheaton, IIl. 60187

STUART S. SMITH, Instr., Adrian College, Adrian, Mich. 49221

FRANCIS T. SMITH, Dir. Lang. Lab., Seton Hall Univ., S. Orange, N. J. 07079

DR. PHILIP D. SMITH, Coord., Penn. For. Lang. Re. Pro., Research Ct., 110 W. Rosedale Ave., West Chester, Penn. 19380

WM. FLINT SMITH, Dir. Lang. Labs., Purdue Univ., Dept. Mod. Lang., Lafayette, Ind. $\mathbf{4 7 9 0 7}$ WILLIAM J. SMITHER, Dir. Lang. Lab., Tulane Univ., New Orleans, La. 70118

EDWARD SNEADE, Coord. For. Lang., Grambling Col., P. O. Box 235, Grambling, La. 71245 LEONARDO SODERMAN, Dir. Lang. Lab., Univ. Concepcion Chile, Casilla 2307, Concepcion, Chile

DAVID G. SPEER, Chrm. Dept. For. Lang., Univ, S. C., Columbia, S. C. 29208

N. G. SPILliOS, Edmonton Pub. Sch. Bd., Edmonton, Alberta, Canada

STATE OF TEXAS, Sam Houston St. Col., For. Lang. Dept., Huntsville, Texas 77340

MRS. M. ANN STEINBERG, Lang. Lab. Dir., 501 Crescent St., New Haven, Conn. 06515

MRS. MARJORIE M. STONER, Exec. Sec., Southwestern at Memphis, Emily Sinpson Courtenay Lang. Ct., Memphis, Tenn. 38112

MARY D. VANDE STREG, Indian Hill Jr., Sr. H. S.. 6845 Drake Rd.. Cincinnati, Ohio 45243 JesuS David SUAREZ, Prof. Sp., Boston Col., 19 Blackthorn Rd., Framingham, Mass. 01701 MR. SEVERIN SWANSON, Wisconsin St. Univ., Oshkosh. Wis. 54901

MARTH H. SWIFT, Lang. Lab. Dir., Morgan Park Acad., 2153 W. 111th St., Chicago, Ill.

MRS. J. M. TANNER, Dir. Super., Univ. Va., Rom. Lang. Dept., 302 Cabell Hall, Charlottesville, Va. 22903

PROF. YVONNE JOAN TAPSON, Presbyterian College, Clinton. S. C. 29325

DR. A. F. TARAS, Audio Lab. Ctr. Dir., Ithaca College, Ithaca, N. Y. 14805

SEITOKU TERUYA, Instr., Univ. Neb., Romance Lang., Lincoln, Neb. 68508

TEXAS A. \& I. UNIVERSITY, Modern Lang., Kingsville, Texas 78363

JEAN R. THFUMA, Lang. Lab. Dir., Univ. Hawaii, Honolulu, Hawaii 96822

DONALDO J. THOMANN, Chrm. Mod. Lang. Dept.,Pacific Union Col., Angwin, Calif. 94508

FREDERICK M. THOMAS, Chrm. For. Lang., Oscar A. Carlson H. S., 30550 W. Jefferson Ave., Gilbraltar, Mich. 48173

BRENDA THOMPSON, Instr., Tri State College, Angola, Indiana

E. FORDYCE THORNE, Dir. Lang. Lab., 7 Houston Rd., Yardley, Penn. 19067

SR. M. TIMONA, SSND, Dir. Lang. Lab., Mt. Mary Col., Milwaukee, Wis. 53222

JON M. TOLMAN, Indiana Univ., Ballentine 851, Bloomington, Ind. 47401

MRS. GERTRUDE M. TRIPP, Dir. Lang. Lab., Elmhurst Col., 190 Prospect Ave., Elmhurst, III. 60126

ELEANOR TAGLIERI, Dir. Lang. Lab., Northeastern Univ., 360 Huntington Aṿe., Boston, Mass. 02115

UNIVERSITY LANG. LAB., Rm. 4, Peterson Hall, 3460 McTavish St., McGil Univ., Montreal 2. Canada

HENRY URBANSKI, Dir. Lang. Lab.. N. Y. St. Univ, at New Paltz, New Paltz. N. Y. 12561

PROF. RAMON J. VALDEZ, 4829 Douglas McArthur N. E., Albuquerque, N. M. 87110

REBECCA M. VALETTE, Dir. Lang. Lab., Boston Col., Chestnut Hill, Mass. 02167

ODILE VANDERGRIFT, Co-Dir., Northern Ill. Univ., Watson Hall, Lang. Lab., DeKalb, Ill.

ROBERT W. VELEY, Chrm. Ger. Dept., Benson Polytechnic Sch., 546 N. E. 12th Ave., Portland, Oregon 97232

DONALD M. VESEY, Dir. Lang. Lab., Seattle Pacific Col., Seattle, Washington 98119

HARRY A. WALBRUCK, Univ. Wis., Racine Center, 1001 S. Main St., Racine, Wis. 53403

S. MARGareT WallaCE, Dir. Lang. Lab., Grove City College, Grove City, Penn. 16127

SALLY WARE, Dir. Lang Lab., 5050 E. St. Strect, Rockford, IIl. 61101

J. M. WATKINS, Dir. Lang. Lab., Middlebury College, Middlebury, Vermont 05753

REV. HAROLD WATSON, OSB, Mod. Lang. Chrm., St. Benedict's Col., Atchison, Kan. 66002 
JACK WATSON, Lang. Lab. Dir., Williams Col., Karl E. Weston Lang. Ctr., Williamstown, Mass. 01267

JOHN A. WATSON, Dir. Lang. Lab., Virginia Union Univ., Richmond, Virginia 23220

DR. HARRY C. WEBB, Audio-Visual Dir., Col. of St. Thomas, 2115 Summit Ave., St. Paul, Minnesota 55101

DONALD B. WEBER, Lang. Lab. Dir., The Univ. of So., Sewanee, Tenn. 37375

DR. ARNOLD L. WEINKAUF, Asst. Prof. Lang., Mich. Tech. Univ., Houghton, Mich. 49931 JOHN W. WELKER, Assoc. Prof., Austin Peay St. Col., Clarksville, Ind. 37040

THE WESTMINSTER SCHOOLS, 1424 W. Races Ferry Rd. NW, Atlanta, Ga. 30327

D. W. WHEETLEY, Dir. of AVA Ed., 194 Boylston St., Lincoln School, Brookline, Mass.

PETER WIESE, 2505 E. 5th St., Apt. 4, Greenville, N. C. 27834

DR. GEORGE W. WILKINS JR., Acting Dir. Lang. Lab., Tulane Univ., New Orleans, La. LARRY WILLIAMS, Adm. Bidg., 1580 Yarrow St., Lakewood, Colo. 80215

ROBERT M. WILLIS, Dir. N. Y. Unir., 435 E. Bidg., Wash. Sq., New York, N. Y. 10003 MRS. T. VADEN WINN, Pearsall H. S., Box 1291, Pearsall, Texas 78061

MARY ISABEL WINSLOW, Pro. Rom. Lang., St. Mary of the Plains Col., Dodge City, Kan. JOE WIPF, Inst. Ger., Univ. S. D., Vermillion, S. D. 57069

WORCESTER POLYTEC. INST., Worcester, Mass. 01609

JUAN DE WYSKOTA, P. O. Box 402, Berry College, Mount Berry, Ga. 30149

J. D. YARBRO, Prof. For. Lang. Dept., U. S. Naval Academy, Annapolis, Maryland 21402 JAMES OWEN YZENBAARD, Supv. Lang. Lab., Western Mich. Univ., Kalamazoo, Mich. PROF. DR. ZIELINSKI, Dir. Inst. F. Erziehungsw D. Rhein-Westf. TH, si A A C H E N, Sandkaulbach 10, Germany

MRS. SUDIE M. ADAMS, Asst. Prof of Sp. \& Port., Baylor Univ., Box 159, Waco, Tex. 76703 SALVAdOR ALVAREZ, Lang. Conslt., Corpus Christi Pub. Sch., 515 N. Carancahua, P. $O$.

Box 110, Corpus Christi, Texas 78401

CECIL E. ATTIG, Supv. Calif. St. Col., Fullerton, 800 N. St., College Blvd., Fullerton, Calif. GILeS AUGer, Asst. Prof., Nasson College, Springville, Maine 04083

WILlIAM H. AUSMAN, Monroe Hall, Rm. 303, George Washington Univ., 2115 G. St., NW Washington, D. C. 20006

MRS. REID E. BAKER, Lang. Lab. Dir., Allegheny College, Meadville, Pa. 16335

CAROIYN BENBOW, Dir. Lang. Lab., Tea. Col., Columbia Univ., Box 66, 525 W. 120th, New York, N. Y. 10027

MRS. VIRA BODNAREK, Lang. Lab. Dir., Chicago City Col., Bogan Campus, 3939 W. 79th St., Chicago, Ill. 60652

DEAN PAULINE FRANCES BOXA, Dean Junior College, Franklin, Mass. 02034

DELIA O. BRANA, Tea. Fr., Forest Hills H. S., (home) 34-10 75th St., Jackson Hgts., N. Y. SISTER ROSE BRENDAN, Barry College, 11300 NE Second Ave., Miami, Fla. 33161

DR. FRANCESCA COBECCHIA, Dir. Lang. Lab., Duquesne Univ., Pittsburgh, Pa. 15219

CARTHAGE COLLEGE LAB. DIR., Kenosha, Wisconsin 53140

VINCENT DeBENEDITIS, Chrm. Lang. Dept., State Col. at Worcester, Worcester, Mass, 01602 REV. R. C. DEPEAUX, St. Norbert College, W. Depere, Wisconsin 54178

BARRY L. ELLIS, 609 E. Main St., Pickney, Mich. 48169

ALVINA E. FANTINI, Dir. FL Dept., Sch. for International Trn., Brattleboro, Vermont 05301 MRS. Alice GATChell, Asst. Prof. Mod. Lang. Dept., Webster Col., 470 E. Lockwood, Webster Groves, Md. 63119

BILlIE GRIGG, Lang. Lab. Sup., El Camino College, El Camino College, Calif. 90506

MRS. KATHLEeN J. GUZEK, Tea. of French, Ontonagon Area H. S., 304 Pennsylvania Ave., Ontonagon, Mich. 49953

PIERIE C. HABER, Dir. Lang. Lab., Jersey City St. Col., Jersey City, N. J. 07305

JACOBINA HARDING, FL, Dept., Chrm., Lang. Lab. Supv., Alamo Hges. H. S., 6900 Broadway, San Antonio, Texas 78209

ADELE B. HERNANDEZ, Instr. of Sp., Univ. of Maine, 96 Flamouth St., Portland, Me. 04103 DIR L. JACKSON, Dir. Lang. Lab., Carleton Univ., Ottawa, Ontario, Canada

SISTER JOAN OF ARC, O. P. Rosary H. S., 8551 Greenfield Ave., Detroit, Michigan 48228 MR. DENNIS KEHOE, Lang. Lab. Dir., Wisconsin St. Univ., Whitewater, Wisconsin 53190 RITA KEHOE, Asst. Lang. Lab. Dir., Wisconsin State Univ., Whitewater, Wisconsin 53190 MR. LINWOOD S. KEMP, Lang. Lab. Technician, Westfield St. Col., Westfield, Mass. 01085 L.ANG. LAB. DIR., Nasson College, Springvale, Maine 04038 
BRO. RICHARD McALliSTER, Spanish Head, Senator O'Connor Col., 1990 Victoria Park Ave., Scarboro, Ontario, Canada

MARTIN M. MAESTAS, 5570 Winchelsou Dr., Normandy, Missouri 63121

SISTER MARIE MAGDALEN, Lang, Lab. Dir., Rosary College, River Forest, Ill. 60305

SISTER MARY CATHERINE, OP Chrm., Dept. For. Lang., Edgewood Col. of the Sacred Heart, 855 Woodrow St., Madison, Wisconsin 53711

MRS. MARTHA R. MILLER, Sec. to Dir., Harvard Univ., Mod. Lang. Ctr., Boysiton Hall G-3, Cambridge, Mass. 02138

SHELBY C. MILLER, Dir. Lang. Lab., Boston Univ., Geddes Lang. Lab. CLA, 725 Commonwealth Ave., Boston, Mass. 02215

MR. ARMANDO PARTINO, Defense Lang. Inst., U. S. Naval Sta., Navy Annex, Anacosta, Washington, D. C. 20390

MR. MAYNARD PLAYFOOT, Asst. Prof, of Romance Lang., Gettysburg College, Gettysburg, Penn. 17325

MISS CONSTANCE L. REID, Instr. German,, Gettysburg Col., Dept. of German, Gettysburg, Pa. 17325

MRS. PATRICIA B. ROSENFIELD, Dir. Lang. Lab., Newton Jr. Col., Washington Park, Newtonville, Mass. 02160

PETER RUNDE, French Dept., Lomira Community Schls., Lomira, Wis. 53048

ELIZABETH F. SCHMITZ, Fr. Lab. and Instr., Sacred Heart Dominican Col,, Holcombe Blvd., Houston, Texas 77021

JUNE V. SIKES, Chrm. For. Lang. Dept., Mission Viejo H. S., Chrisanta Dr., Mission Viejo, Calif. 92675

MR. SEVERIN A. SWANSON, Dir., Wisconsin St. Univ., Oshkosh, Wisconsin 54901

ELEANOR TAGLIERI, Instr. Northeastern Univ., 32 Gould St., Boston, Mass. 02132

BRO. JEROME TUPA, OSB, St. John's Univ., Lang. Lab. Dir., Collegeville, Minn. 56321

MR. EDUARDo E. VARGAS, Asst. Prof. Sp., Missouri Western College, Tenth and Edmond Sts., St. Joseph, Missouri 64501

NANCY J. WEINGARTEN, Harvard Univ., Grad. Sch. of Ed., 175 Beech St., Belmont, Mass. KARL WIDMAIER, Mod. Lang. Instr., Atlantic Union College, South Lancaster, Mass. 01561 MRS. J. M. YALDEN, St. Patrick's College, Carleton Univ., 4 Ava Rd., Ottawa 7, Ontario, Canada.

NOTE: Some names arrived at the Publication Center too late to be included on this list. Please accept our apologies for omission or errors. 